\title{
Theory and proposal for a quantum-degenerate electron source
}

\author{
M. Zolotorev, E. D. Commins, ${ }^{*}$ and F. Sannibale \\ Ernest Orlando Lawrence Berkeley National Laboratory, \\ One Cyclotron Road, Berkeley, California 94720, USA
}

(Dated: September 11, 2006)

\begin{abstract}
We propose a pulsed electron source capable of a $6 \mathrm{D}$ brightness orders-of-magnitude greater than that of existing sources. It could deliver average current up to $0.5 \mathrm{pA}$ and achieve an emittance approaching the quantum limit of one Compton wavelength in each spatial dimension. It could be employed to advantage in electron microscopy, inverse photo-emission, precision low-energy scattering experiments, and electron holography. This source could make possible pump-probe experiments with Angstrom spatial and sub-picosecond time resolution. Here we describe basic concepts of the source, including analysis of main issues that must be addressed for its successful construction and operation. We have begun an experiment to demonstrate its essential features.
\end{abstract}

PACS numbers: 29.25.Bx, 41.75.Fr, 32.80.Rm, 41.85.Ct, 29.27.Bd

*Also at Department of Physics, University of California, Berkeley, California 94720. 
We propose a pulsed electron source with brightness approaching the quantum mechanical limit [1]. It has the potential for many useful applications in electron microscopy, inverse photo-emission, and low energy electron scattering experiments. The source makes use of alkali atoms in an atomic beam. (We illustrate our ideas with Cs, but other alkalis, for example K, might be employed). Each cycle of the device begins with a laser pulse that excites a single Cs atom, on average, to a band of high-lying Rydberg nP states. The radial motion of the resulting shell-like valence-electron Rydberg wave packet is nearly classical. When the electron reaches apogee, an electric field pulse is applied that ionizes the atom and accelerates the electron away from its parent ion. The electron bunch thus generated in a train of cycles can occupy a phase volume near the quantum limit and possess very high brightness. Thus the source may be employed to observe coherence effects, e.g. electron holography. As in any coherence experiment involving electrons, interference occurs between different paths over a single electron wave-packet, and successive wave packets are mutually incoherent. However if these packets are similar enough to one another, an interference pattern with significant visibility emerges from the train of pulses. We shall see that this can be achieved with the proposed source.

We first review the usual description of electron bunches. [We assume that all electrons are non-relativistic in the center of momentum (CM) frame. In our application this is the frame where the average position and momentum of the Rydberg atom nuclei vanish.] The dimensionless differential volume in phase space is:

$$
d \Gamma=\frac{d x d p_{x} d y d p_{y} d z d p_{z}}{(2 \pi \hbar)^{3}}=\frac{d x d \beta_{x} d y d \beta_{y} d z d \beta_{z}}{(2 \pi)^{3} \lambda_{c}^{3}}
$$

where $\beta_{x, y, z}=v_{x, y, z} / c, c$ is the velocity of light, and $\lambda_{c}=\hbar / m_{e} c=3.86 \times 10^{-11} \mathrm{~cm}$ is the electron Compton wavelength. The rms emittances $\epsilon_{i}$ are:

$$
\begin{aligned}
\epsilon_{i}^{2}=\left(<r_{i}^{2}>-\right. & \left.<r_{i}>^{2}\right)\left(<\beta_{i}^{2}>-<\beta_{i}>^{2}\right) \\
- & \left(<r_{i} \beta_{i}>-<r_{i}><\beta_{i}>\right)^{2}
\end{aligned}
$$

with $i=x, y, z[2]$, and where $<\ldots>$ means average over the ensemble. In the C.M. frame, we have $<r_{i}>=0,<\beta_{i}>=0$. If in addition there are no correlations between $r_{i}$ and $\beta_{i}$, $<r_{i} \beta_{i}>=0$. Then (2) becomes:

$$
\epsilon_{i}=\sqrt{<r_{i}^{2}><\beta_{i}^{2}>}
$$


Frequently we can assume that the distribution function $f=j J$ in phase space is Gaussian, where

$$
j(x, y, z)=\frac{\exp \left[-\left(x^{2} / 2 \sigma_{x}^{2}+y^{2} / 2 \sigma_{y}^{2}+z^{2} / 2 \sigma_{z}^{2}\right)\right]}{(2 \pi)^{3 / 2} \sigma_{x} \sigma_{y} \sigma_{z}}
$$

and

$$
J\left(\beta_{x}, \beta_{y}, \beta_{z}\right)=\frac{\exp \left[-\left(\beta_{x}^{2} / 2 \eta_{x}^{2}+\beta_{y}^{2} / 2 \eta_{y}^{2}+\beta_{z}^{2} / 2 \eta_{z}^{2}\right)\right]}{(2 \pi)^{3 / 2} \eta_{x} \eta_{y} \eta_{z}} .
$$

Then (3) becomes $\epsilon_{i}=\sigma_{i} \eta_{i}$, and the dimensionless volume in phase space occupied by the electron beam is $\Gamma=\epsilon_{x} \epsilon_{y} \epsilon_{z} / \lambda_{c}^{3}$. Let $N_{e}$ be the actual number of electrons. The degeneracy parameter $\delta_{F}$ is:

$$
\delta_{F}=N_{e} / \Gamma=\lambda_{c}^{3} N_{e} /\left(\epsilon_{x} \epsilon_{y} \epsilon_{z}\right)=\lambda_{c}^{3} B,
$$

where $B=N_{e} / \epsilon_{x} \epsilon_{y} \epsilon_{z}$ is the '6D-brightness'. For electrons of given spin polarization, the Pauli principle requires $\delta_{F} \leq 1$. For all electron bunches generated by conventional sources, $\delta_{F}<<1$, and the emittances $\epsilon_{x, y, z}$ are dominated by stochastic effects, such as finite source spatial dimensions and temperature, electron-electron Coulomb heating [3], etc. For example, field emission electron sources operate in the classical regime, and those of the most advanced design achieve $\delta_{F} \sim 5 \times 10^{-6}-5 \times 10^{-5}[4,5]$.

However, we shall prepare alkali atoms one-by-one in a band of high-lying Rydberg states, by pulsed laser excitation of a slow atomic beam in a very small laser-atom interaction volume $V$. This is done so that at apogee each valence electron has very nearly the same spatial wave-function, which is shell-like with radius $R$ and thickness $\Delta R<<R$. In this case we must expand the meaning of the degeneracy parameter $\delta$ and express it in quantum mechanical terms [6]. We now do this and show how $\delta$ goes over to $\delta_{F}$ as given by (6) in the limit of sufficiently large $V$ and high atomic beam temperature.

In the CM frame, consider two identical atoms, where one nucleus is displaced from the other by distance $\vec{u}$, and has 'velocity' $\vec{\beta}=\vec{p} / m c$ with respect to the other. [We ignore the very small uncertainty $\Delta u \Delta \beta \sim \hbar / M c$ where $M$ is the Cs atomic mass]. We assume that $\vec{u}$ and $\vec{\beta}$ have normal probability distributions about their respective origins, as in (4) and (5). Let the normalized valence electron wave-function at apogee be $\psi(\vec{r})$ when $\vec{u}=\vec{\beta}=0$. The anti-symmetrized two-electron wave function is:

$$
\begin{array}{r}
\Psi\left(\vec{r}_{1}, \vec{r}_{2}\right)=2^{-1 / 2}\left[\psi\left(\vec{r}_{1}\right) \psi\left(\vec{r}_{2}-\vec{u}\right) \exp \left(i \vec{p} \cdot \vec{r}_{2} / \hbar\right)\right. \\
\left.-\psi\left(\vec{r}_{2}\right) \psi\left(\vec{r}_{1}-\vec{u}\right) \exp \left(i \vec{p} \cdot \vec{r}_{1} / \hbar\right)\right]
\end{array}
$$


The corresponding density matrix is:

$$
\begin{array}{r}
\rho\left(\vec{r}_{1}, \vec{r}_{2}, \vec{r}_{1}{ }^{\prime}, \vec{r}_{2}{ }^{\prime}\right)= \\
\int j(\vec{u}) d^{3} \vec{u} \int J(\vec{\beta}) d^{3} \vec{\beta} \cdot \Psi\left(\vec{r}_{1}, \vec{r}_{2}\right) \Psi^{*}\left(\vec{r}_{1}{ }^{\prime}, \vec{r}_{2}{ }^{\prime}\right) .
\end{array}
$$

The trace of the density matrix is $\operatorname{tr}(\rho)=1-\delta$, where

$$
\begin{array}{r}
\delta=\int j(\vec{u}) d^{3} \vec{u} \int J(\vec{\beta}) d^{3} \vec{\beta} \\
\times\left|\int \psi\left(\vec{r}-\frac{\vec{u}}{2}\right) \psi^{*}\left(\vec{r}+\frac{\vec{u}}{2}\right) \exp \left(i \frac{\vec{\beta} \cdot \vec{r}}{\lambda_{c}}\right) d^{3} \vec{r}\right|^{2} .
\end{array}
$$

For simplicity we assume $\sigma_{x}=\sigma_{y}=\sigma_{z} \equiv \sigma$ and $\eta_{x}=\eta_{y}=\eta_{z} \equiv \eta$. When $\sigma=\eta=0$, $\delta=1$. Also, it can be shown that when $\sigma>>R, \eta>>\lambda_{c} / \Delta R$, then $\delta \rightarrow\left[\lambda_{c} /(\sigma \eta)\right]^{3}$. Thus $\delta$ becomes equal to $\delta_{F}$ in the (classical) limit of large $\sigma, \eta$, and (9) is a suitable definition of the degeneracy parameter. The 2-particle density matrix (8) is equivalent to a two-particle distribution function [7] but one can also consider $N>2$ particles. However, the resulting generalization of (9) behaves as follows in the limit of large $\sigma$ and $\eta: \delta_{N>2} \rightarrow\left[\lambda_{c} /(\sigma \eta)\right]^{3(N-1)}$ which is negligible compared to $\delta$ in $(9)$.

We now consider the characteristics of $\delta$ for our proposed source, (see Fig. 1). An atomic beam effusing from an oven at temperature $\sim 500 \mathrm{~K}$ can be collimated to reduce the transverse beam temperature to $\sim 5 \mathrm{~K}$, and transverse cooling using the $6^{2} S_{1 / 2}-6 P_{3 / 2}$ Cs resonance line can be employed to further reduce this temperature to $\sim 0.01 \mathrm{~K}$. Also, laser excitation along the beam axis can be used to select a narrow band of longitudinal velocities. Thus excited atoms could have a thermal energy spread $\Delta E \leq 10^{-6} \mathrm{eV}$, and if the valence electrons were optically excited to ionization threshold and we could ignore subsequent space-charge heating, the electron energy spread could be $\delta E=\left(m_{e} / M_{C s}\right) \Delta E \leq 10^{-11}$ $\mathrm{eV}$. However, if many atoms were ionized in a given pulse, space-charge interactions would result in an electron temperature given by $k T \sim e^{2} n_{e}^{1 / 3}$ where $n_{e}$ is the electron density [8]. For example, given a laser-atom interaction volume $\sim 10^{-9} \mathrm{~cm}^{3}$ and $n_{e} \sim 10^{10} \mathrm{~cm}^{-3}$, so that only $\sim 10$ electrons are generated simultaneously, space-charge interaction would yield $\delta E \sim 10^{-4} \mathrm{eV}$. This heating is avoided by exciting one atom at a time, on average, to a very high lying band of $n P$ Rydberg states (for example $\bar{n} \sim 800, \Delta n \sim 50$ ), with 3 mutually perpendicular laser beams: [L1: cw, $\left.852 \mathrm{~nm}\left(6 S_{1 / 2}-6 P_{3 / 2}\right)\right]$; [L2: cw, $1.47 \mu \mathrm{m}\left(6 P_{3 / 2}-7 S_{1 / 2}\right)$ ]; [L3: pulsed, $\left.777 \mathrm{~nm}\left(7 S_{1 / 2}-n P\right)\right]$. These laser beams intersect the atomic beam in a small 


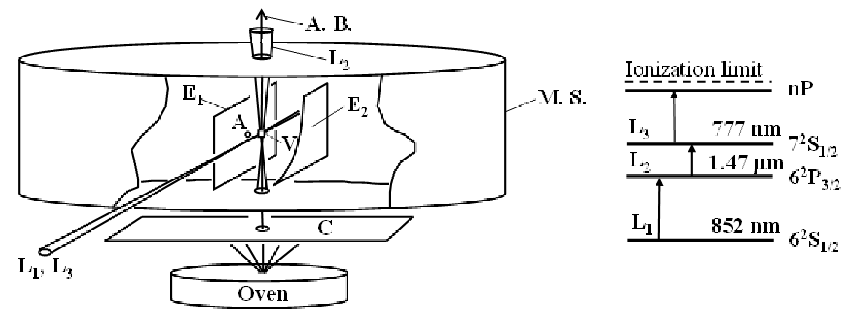

FIG. 1: Schematic diagram of source (not to scale). C: atomic beam collimator; M.S.: magnetic shield; $\mathrm{L}_{1}, \mathrm{~L}_{2}, \mathrm{~L}_{3}$ : Laser beams; V: laser-atom interaction region; $\mathrm{E}_{1}, \mathrm{E}_{2}$ : plane electrodes; A.B.: atomic beam; A: electron extraction aperture. Transverse cooling laser beams not shown.

interaction volume $V \sim(10 \mu m)^{3}$ midway between two plane parallel electrodes E1, E2 with separation $\sim 1 \mathrm{~cm}$. The resulting valence-electron Rydberg wave packet forms a spherical shell that expands radially in Kepler-like motion, with half-period $T=\left(\pi \hbar^{3} \bar{n}^{3}\right) /\left(m_{e} e^{4}\right)$. (We choose $\bar{n} \sim 800$ as an example because in this case $T \sim 40$ ns, which makes possible a range of pulsed laser bandwidths convenient for chirping. However, $\bar{n} \sim 600$ might also be practical). The oscillator strengths for $7 S-n P_{3 / 2}$ transitions are much larger than for Cs $7 S-n P_{1 / 2}$ when $n \geq 15$ [9]. Taking into account this spin-orbit effect [10] and assuming that the $777 \mathrm{~nm}$ photons are linearly polarized along $z$, the probability density of the Rydberg wave packet is proportional to $1+3 \cos ^{2} \theta$, where $\theta$ is the angle between the $z$ axis and the electron position vector $\vec{r}$.

One wants the shell width $\Delta R$ at apogee to be reasonably large, because this relaxes restrictions on $V$. Also it is desirable that the radial part $F$ of the wave function describing the wave packet at apogee be real (apart from an arbitrary overall phase), so that the radial probability current density vanishes everywhere. These goals are achieved by suitable chirping of the $777 \mathrm{~nm}$ laser pulse, the conditions for which are derived as follows. First, it is convenient to express $F$ as a function of $r / R$ and $t / T$, where $R \cong 2 \bar{n}^{2} a_{0}$ is the classical outer turning point for $\bar{n}$. (For $\bar{n}=800, R=1.28 \cdot 10^{6} a_{0} \cong 68 \mu \mathrm{m}$, where $a_{0}$ is the Bohr radius). At any time $t$ after the laser pulse we can express $F(r / R, t / T)$ in terms of the radial eigenstates $u_{n}(r / R)$ of the atomic Hamiltonian:

$$
\begin{array}{r}
F(r / R, t / T)=\sum c_{n} u_{n}(r / R) \exp \left(-i \omega_{n} t\right)= \\
\sum c_{n} u_{n}(r / R) \exp \left[i \pi \bar{n}^{3} /\left(2 n^{2}\right) \cdot t / T\right]
\end{array}
$$


where the $c_{n}$ are constants and $\omega_{n}=E_{n} / \hbar$. Equating (10) to $F(r / R, 1)$, (assumed real), at $t=T$, and making use of the orthonormality of the $u_{n}$, we obtain:

$$
c_{n}=\exp \left(-\frac{i \pi}{2} \frac{\bar{n}^{3}}{n^{2}}\right) \int u_{n}^{*} F\left(\frac{r}{R}, 1\right) r^{2} d r .
$$

Since $F(r / R, 1)$ varies very slowly with respect to $r$ compared to the $u_{n}$ for $n>>1$, one can show that (11) yields:

$$
c_{n} \cong(-1)^{n} \exp \left[-i \pi \bar{n}^{3} /\left(2 n^{2}\right)\right] F\left(n^{2} / \bar{n}^{2}, 1\right) .
$$

Also, from first-order time dependent perturbation theory the coefficients $c_{n}$ are related to the electric field amplitude $g(t / T)$ of the $777 \mathrm{~nm}$ laser pulse by:

$$
c_{n}=\frac{\text { const }}{n^{3 / 2}} \int \exp \left[\frac{i \pi}{2} \bar{n}\left(1-\frac{\bar{n}^{2}}{n^{2}}\right) \frac{t}{T}\right] g(t / T) d t,
$$

where the factor $n^{-3 / 2}$ is proportional to the dipole matrix element connecting the $7 \mathrm{~s}$ and np states, when $n>>1$. Comparing (12) and (13) and using the Fourier integral theorem we find that:

$$
\begin{array}{r}
g(t / T)=\text { const } \int_{-1}^{1} f(x) \\
\times \exp \left\{-i \pi \bar{n}\left[(1-x)^{-1 / 2}+(1-x) / 2+x t /(2 T)\right]\right\} d x
\end{array}
$$

where $f(x)=(1-x)^{-3 / 4} F\left[(1-x)^{-1}, 1\right]$. Since $f(x)$ varies slowly compared to the rapidly oscillating phase factor in the integrand of (14), this integral can be evaluated with sufficient accuracy by the stationary phase approximation [11]. One thus obtains $g(t / T)=A(t / T) \exp [i \phi(t / T)]$ where

$$
A(t / T) \cong \mathrm{const} \cdot(1-t / T)^{-1 / 3} F\left[(1-t / T)^{2 / 3}, 1\right]
$$

and conversely,

$$
F(r / R, 1)=(r / R)^{1 / 2} A\left[1-(r / R)^{3 / 2}\right]
$$

while

$$
\phi(t / T)=-\pi \bar{n}\left[(3 / 2)(1-t / T)^{1 / 3}+t /(2 T)\right] .
$$

The analysis leading from (10) to (17) has been checked by numerical integration of Schroedinger's time-dependent equation for excitation of a Cs atom by a laser pulse given by 


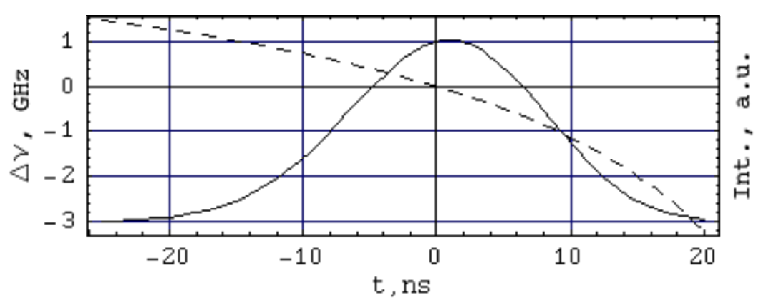

FIG. 2: Laser pulse intensity (solid curve) and change in frequency (dashed curve) for chirped pulse described by Eqs. (15) and (17)

$(15,17)$ assuming a definite $F(r / R, 1)$, and subsequent evolution to apogee. The calculation reproduces the initial choice of $F(r / R, 1)$ to very good accuracy regardeless of the form of $F$ within wide limits, so long as $\phi$ is given by (17). Thus we only need to specify the overall width of $F$ (hence of $A$ ) and the form of $\phi$ in order to determine $\delta$. In Fig. 2 we plot the pulse intensity $[A(t)]^{2}$ and the pulse frequency $\nu=$ const $-1.03 \times 10^{10}\left[\frac{3}{2} \frac{(1-t / T)^{1 / 3} T}{t}+\frac{1}{2}-\frac{3 T}{2 t}\right]$ Hz obtained from (17), assuming $F(r / R, 1)$ is Gaussian:

$$
F(r / R, 1)=\text { const } \cdot \exp \left[-(r-R)^{2} /\left(4 \Delta R^{2}\right)\right]
$$

with $\bar{n}=800$ and $\Delta R=0.12 R$.

One can show that when $F(r / R, 1)$ is given by (18), formula (9) for $\delta$ with the Gaussian distributions (4), (5) can be expressed as follows, assuming $\sigma<<R$ and $\eta \Delta R<<1$ :

$$
\delta=\int_{0}^{1} \frac{\exp \left(-2 \eta^{2} R^{2} x\right)}{\sqrt{1+\frac{\sigma^{2}}{2 \Delta R^{2}}+\frac{\sigma^{4}}{4 \Delta R^{4}} x(1-x)}} d x .
$$

In Fig. 3 we plot curves of constant $\delta$ from (19) for two cases: a) The chirped laser pulse described by (15), (17) with (18), $\bar{n}=800, \Delta R=0.12 R$; b) An unchirped Gaussian laser pulse amplitude with time dependence $\exp \left(-t^{2} / 4 \tau^{2}\right)$. We choose $\tau=1$ ns because this gives a wave packet with minimum uncertainty $\Delta r \Delta p_{r}$ at apogee as a function of $\tau$ for $\bar{n}=800$. Here, $\Delta R \sim 0.02 R$. This unchirped laser pulse yields a radial wave function at apogee with an $r$-dependent phase factor, and thus a radial probability current density that does not vanish identically. It can be shown that this results in $\delta$ described by (19) where $\sigma$ in the integrand is replaced by $\sigma_{\text {eff }} \sim \sqrt{2} \sigma$.

It appears practical to achieve $\sigma \sim 10 \mu \mathrm{m}$ and $\eta c \sim 100 \mathrm{~cm} / \mathrm{s}$ in a real apparatus. Assuming this, Fig. 3 shows that for case a) $\delta \sim 0.6$, while for case b) $\delta \sim 0.03$. This reveals 


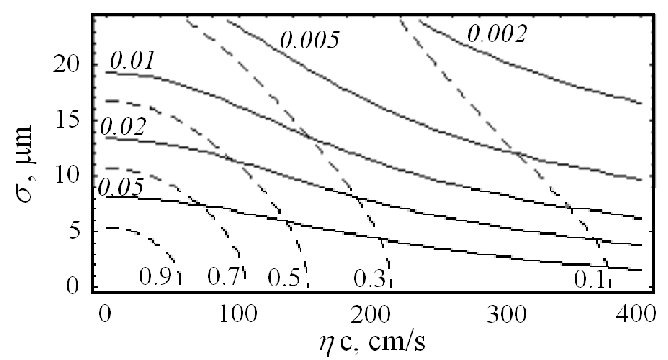

FIG. 3: Curves of constant $\delta$ in the $\eta \sigma$ plane, calculated from Eq. (19). Dashed curves: case a) chirped with $\Delta R=0.12 R$. Solid curves: case b) no chirp, $\Delta R \sim 0.02 R$

the large advantage of chirping and suggests that with it, substantial coherence effects can be realized.

We next discuss various important considerations for any real experimental device. At apogee, an electric field pulse with peak value $\mathcal{E}_{0}$ and $\mathrm{FWHM}=\tau_{0}$ must be applied to ionize the atom and accelerate the electron. $\mathcal{E}_{0}$ and $\tau_{0}$ should satisfy the following criteria: $\int \mathcal{E} d t$ should be the same for all electrons regardless of $\theta$ at apogee; the acceleration should be rapid enough that Coulomb interaction between the electron and residual $\mathrm{Cs}^{+}$ion is minimal; and the final electron energy $E$ should be sufficiently large $(\sim 1-10 \mathrm{eV})$ that electron-optical aberrations on passing through subsequent apertures are not too serious. Typical acceptable values are $\mathcal{E}_{0}=100 \mathrm{~V} / \mathrm{cm}, \tau=0.5 \mathrm{~ns}$. Because the electrons are close to the quantum degenerate limit, $\Delta E \Delta t \sim \hbar$ where $\Delta t$ is the spread in electron arrival times. By a 'rotation' in phase space, it is possible to vary $\Delta E$ at the expense of $\Delta t$ for diverse applications. For example, in electron microscope and holographic experiments, a very small $\Delta E$ is desirable $\left(\Delta E \sim 10^{-8} \mathrm{eV}\right.$, which corresponds to $\Delta t \sim$ time between pulses $)$, while for pump-probe experiments with sub-picosecond resolution, $\Delta E \sim 10^{-3} \mathrm{eV}$ is adequate.

For a Rydberg atom with principal quantum number $n$, the electric field required for spontaneous ionization is $\mathcal{E}_{I}=e /\left(16 a_{0}^{2} n^{4}\right) \sim 8 \times 10^{-4} \mathrm{~V} / \mathrm{cm}$ for $n=800$. Such a field is generated by an electronic charge at a distance $s=0.014 \mathrm{~cm}$. Therefore we must ensure that when the next laser pulse occurs, the residual ion from the present pulse is at a distance $s^{\prime}>>s$ from the interaction region. To achieve this, after the acceleration pulse another 'ion clearing' electric field pulse must be applied to remove the remaining Cs ion from the interaction region. Because the ion is massive, the clearing pulse must have duration $\sim 100$ 
ns if its amplitude is $\sim 1 \mathrm{kV} / \mathrm{cm}$. In this case, a cycle repetition rate $\sim 3 \mathrm{MHz}$ can be achieved. At an average of one electron per pulse, this gives an average source current of $0.5 \mathrm{pA}$.

It will be necessary to reduce stray electric fields in the interaction region to a level $\mathcal{E}_{S}<<$ $\mathcal{E}_{I}$; that is to the level $\mathcal{E}_{S} \leq 10^{-4} \mathrm{~V} / \mathrm{cm}$. This is feasible, as has been demonstrated by the results of earlier experiments on Rydberg states [12]. Stray magnetic fields $B$ can also cause undesirable perturbations that degrade brightness unless the electron radius of curvature is much greater than $R$. To achieve this one must reduce the ambient magnetic field to a level $\leq 1 \mathrm{mG}$. This can be done by enclosing the interaction region in an appropriate magnetic shield. Care must also be taken to avoid generation of photo-electrons by absorption of stray laser photons on electrode surfaces.

Collisions between Rydberg atoms of interest and ground state atoms as well as molecules are unlikely to cause serious difficulties. We estimate the probability $w$ for a scattering in the time $T=40$ ns between the laser pulse and the acceleration pulse to be $w<<n_{0} \sigma_{\max } u T \sim$ $2 \times 10^{-5}$ where $u \sim 2 \times 10^{4} \mathrm{~cm} / \mathrm{s}$ is the mean relative velocity of the Rydberg atom and ground state atom [13]. The cross-sections for collisions of the Rydberg atom with $\mathrm{Cs}_{2}$ and with background gas molecules are undoubtedly large, but the number densities of these molecules will be so small (with proper design of the atomic beam source and in ultra-high vacuum) that they should not present serious problems. We are encouraged by the results of previous alkali Rydberg atom experiments done in rather rudimentary vacuum conditions, which achieved principal quantum numbers $n \sim 1000[14,15]$.

In conclusion, the proposed source would be a first step toward a novel low-energy scanning electron microscope with current density on the sample of several $\mathrm{kA} / \mathrm{cm}^{2}$ and Angstrom resolution. Such resolution would be achieved for two main reasons: chromatic aberration is minimized by extremely small phase-space volume, and the well-defined time structure of the electron beam allows use of focusing or defocusing time-dependent fields, which implies the possibility of positive or negative spherical aberration. The latter can also be compensated by axial symmetric lenses. The source would also extend applications for electron beams to energy exchange with $\mu \mathrm{eV}$ accuracy in inelastic atomic and molecular scattering, and to new ways of investigating chemical reactions and dynamics on a picosecond time scale using pump-probe techniques. Finally, the feasibility of significant phase coherence opens the possibility of electron holography and other interferometric experiments. 
We thank J. Byrd, S. Chu, D. Colomb, P. Denes, G. Huang, Z. Hussain, S. Heifets, J. Krupnik, G. Lebedev, S. Lidia, J. Oneill, D. Robin, K. Robinson, R. Schoenlein, R. Vogel, and W. Wan, who have provided very valuable discussions as well as scientific and technical support. This work was supported by the Director, Office of Science, of the U.S. Department of Energy under Contract DE-AC02-05CH11231.

[1] M. Zolotorev, et al., Proc. 2005 Particle Accelerator Conf., May 2005, Knoxville TN.

[2] C. Lejeune, J. Aubert, Applied Charged Particle Optic, Advances in Electronics and Electron Physics, A. Septier Editor, Supp. 13 A (1980).

[3] See for example: P. Kruit and G.H. Jansen, Handbook of Charged Particle Optics, CRC Press, Cleveland, Ohio, 1997, pp. 275-319.

[4] J. C. Spence, M. R. Howells, Ultramicroscopy 93, 213 (2002).

[5] N. deJonge, et al, Phys. Rev. Lett. 94, 186807 (2005).

[6] See for example: P. Kruit, M. Bezuijen, and J.E. Barth, J. Appl. Phys. 99, 024315 (2006).

[7] R. P. Feynman, Statistical Mechanics, Benjamin-Cummings, Reading, MS,1972, p. 65.

[8] B. J. Claessens et al., Phys. Rev. Lett. 95, 164801 (2005).

[9] D. Norcross, Phys. Rev. A 7, 606 (1973).

[10] H. Kaminski, J. Kessler, and K. J. Kollath, Phys. Rev. Lett. 45, 1161 (1980).

[11] J. D. Jackson, Classical Electrodynamics, 3rd Ed., Wiley, NY, 1998, p.338.

[12] J. Neukammer, et al., Phys. Rev. Lett. 59, 2947 (1987).

[13] T. F. Gallagher, Rydberg Atoms, Cambridge, NY, 1999, Chapter 11.

[14] M. T. Frey, et al., Rev. Sci. Instrum. 64, 3649 (1993).

[15] M. T. Frey, et al., Phys. Rev. Lett. 75, 810 (1995). 